\title{
DOCUMENT DIGITALIZATION THROUGH USE OF CLOUD COMPUTING TECHNOLOGY
}

\author{
Smita Agade, Shivani Gokhe, Pallavi Chilamkar, Ujwala Gurnule \\ Prof. S. D. Zade \\ Department of Computer Science and Engineering \\ Priyadarshini Institute Of Engineering And Technology, Nagpur.
}

\begin{abstract}
A paperless office, also called a paperfree office, is a work environment which uses minimal physical paper and instead uses primarily digital documents. A paperless employee is a worker who has eliminated or greatly reduced the use of paper in the workplace. The process of converting paper files into electronic files is known as digitization. Even today, a large number of organizations collect data using paper forms. However, it can be difficult to aggregate, and analyse the data collected using paper forms. Better management and processing of documents is indispensable to improving customer experience. The idea of an entirely paperless office has existed since personal computers became the basis of the modern workplace. Despite the prevalence of electronic documents and email, most organizations still rely on paper documents. There are many benefits to going paperless, from saving resources to boosting security. Document Digitalization System is an essential approach that should be manage well to ensure an effective and faster overall working process in an organization .Hardcopy document has been one of the items that most organization need to manage in safe and secure manner due to high dependency on most of their working procedure especially in government organization hence we proposed new framework using cloud computing technology to improve the weakness of the existing Document management procedures in a government organizations . Government organization are utilizing a paperless documentation method to save their overall running cost as well as reducing the need to store massive amount of physical papers. Government departments are still using paper based documentation. This is because of the inefficient security system. Benefits of Document Digitalization are Saves Time, Saves Space, Eases Transfer of Information, Boosts Security, Get Access Anytime, Anywhere, Find Information Faster, Bring Documents Together.
\end{abstract}

Keywords: Document Management System, Information Retrieval.

\section{INTRODUCTION}

The idea of paperless governance, and an aim to make life simpler for user, the government will now be accepted document when present in digital form. Document Digitalization securely save copies of all their documents. It is a platform for issuance and verification of documents and certificates in a digital format, thereby eliminating the use of physical documents. user who sign up for a Digital account get a dedicated cloud storage space that is linked to their account. Government departments and organisations which are registered with Digital account can push electronic copies of documents and certificates issued by them, directly into the user account. User can also upload scanned copies of their documents in their accounts. All physical documents can then be kept in the safety, minimising the chance of ever losing them, or damaging them. Issued documents are e-documents issued by various government agencies in electronic format directly from the original data source and the URI (link) of these documents is available in the issued documents section. Whereas uploaded documents are those e-documents uploaded directly by the user. In Document digitilization only admin can add another admin/ employee for accessing other document by giving their log in information. Admin can search document of user by their Name, Date Of Birth, Contact number. Employee can upload physical document of existing users by creating their account and convert them into digital format. In Document Digitilization new user create their account by filing their application form and upload their documents.

\section{OBJECTIVE}

- Backup and Security Data centers are staffed $24 \times 7$ by trained security guards, and access is authorized strictly on privilege basis. We routinely back up your data on Document Digitalization according to best backup guidelines for any disaster or contingency with the most secure methods. 


\section{International Journal of Engineering Applied Sciences and Technology, 2020 Vol. 4, Issue 10, ISSN No. 2455-2143, Pages 260-262 \\ Published Online February 2020 in IJEAST (http://www.ijeast.com)}

- This saves your money on things like Consultant and maintenance fees, servers, hardware and power charges, and installation charges. Document Digitalization offers almost no servers and hardware upkeep and ultimately the staff needed to maintain these are considerable low in numbers.

- Efficiency And Productivity Users can collaborate around content with sharing of folders and files, post comments on various Document activities, stay informed with automated notifications and alerts and trigger workflows for team members. Working with teams outside your organization more often involves sending documents over courier and other means of Logistics or even emailing attachments..

- A digital document management system helps users to access the required data quickly and precisely, thereby enabling better customer service.

- Digital documents are saved in secured environments (servers, databases, encryption etc.), and can be accessed only by authorized users, which provides more security compared to a paper-based system, where a misplaced or mishandled document is a common problem.

Hone Hautls Serices Tean Corbath

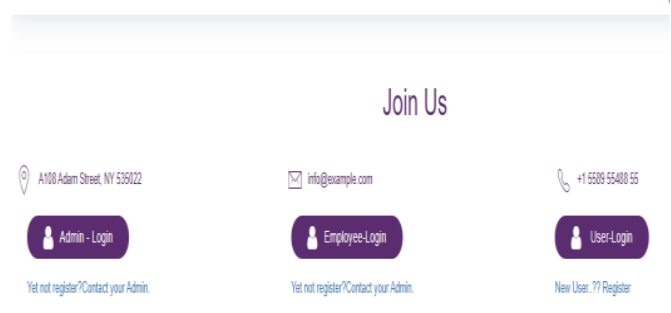

Fig.1.Login Page

There are three modules in this web application which are as:

1. Admin Login Module:

Admin has special powers of giving different user id and password to each employee. Admin can handle update or remove the data of user. Admin can search document of user by their Name, Date Of Birth, Contact number as per their requirement.

\section{Document access}

Document Digitalization is deployed as Software as a Service for you, optimized to run over the Internet and accessible via many web browsers. Document
Digitalization Business lets you accesses your files faster, easier and from anywhere. This proposition of accessing documents also enables efficient collaboration when you are traveling.

Proposed System

An Document Digitalization system is a software system for organizing and storing different kinds of documents. This type of system is a more particular kind of document management system, a more general type of storage system that helps users to organize and store paper or digital documents. Increased customer satisfaction and shorter time-tomarket by better business process. Greater cost savings Workflow Automation processes. Greater employee satisfaction as collaboration and best practices are improved. Implement quickly and easily. No coding is required. Electronic Forms: Easy and Quick eForms set-up. 4 Standard workflows available. Customise as per your requirements. Get your team engaged in your daily processes and ensure maximum productivity. This solution enables authorised users to access, create, search, order, retrieve and edit their inventory from their desktop. We offer customised data entry screens to enforce crucial data capture and retention dates. Powerful and friendly search functionality enables quick and easy query of the record centre database, ensuring that you remain in control.

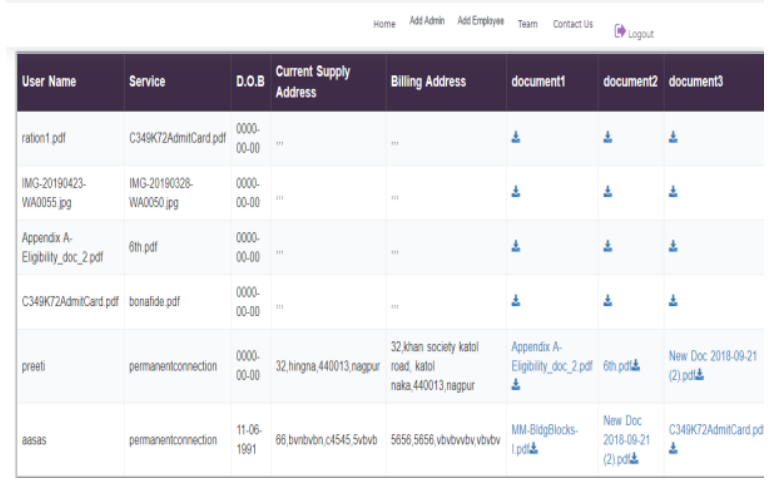

Fig.2.Admin Dashboard page

2. Employee Log In module

Employee can upload physical document of existing users by creating their account and convert them into digital format.

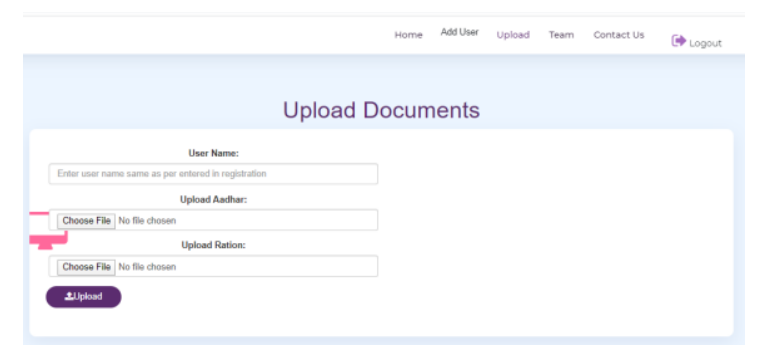


Fig.3.Employee Dashboard page

\section{User Login Module}

new user create their account by filing their application form and upload their document. user can edit their documents and upload new documents.

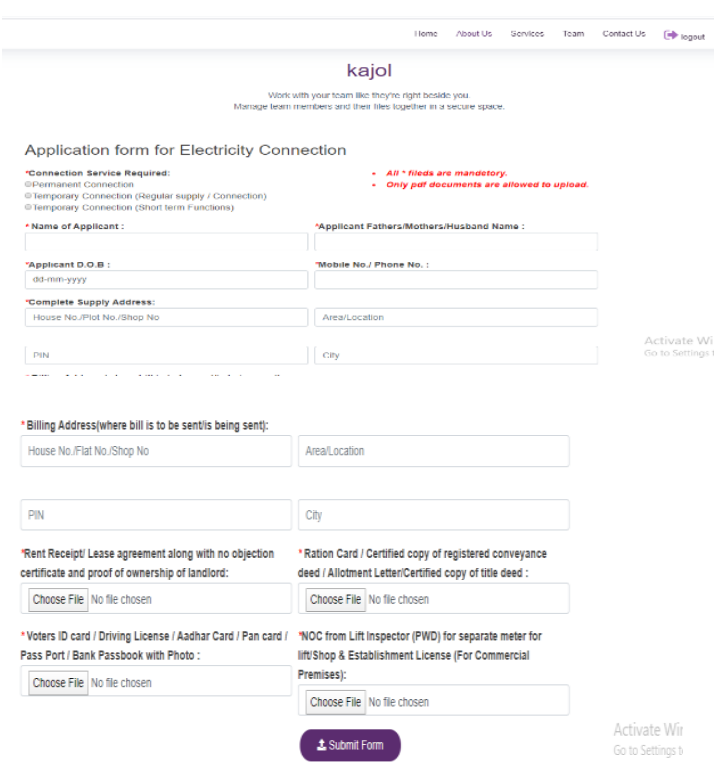

Fig.4.User Dashboard page

Document storage:

Cloud storage offers multiple benefits for individuals and businesses, but sometimes you don't need a big enterprise solution for big data but simply a central repository for key business files and documents .

This is especially to serve as a simple backup for disaster recovery of all your files, folders, such as documents and images, so that in the event of a hard drive failure you can easily recover them from another PC or other device. This can also help free up space in busy offices where saving data across multiple machines can be impractical.

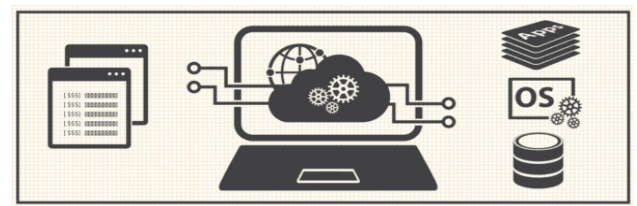

Fig.5.Cloud Storage

\section{CONCLUSION}

In this paper we are trying to introduce an website for Electricity department. This website will help authorised person to access document of user and employer can upload the existing paper document of user into digital formate and new user able to upload there documents and access any time and from anywhere and only authorised person will able to see document of all user. this paper is for converting physical documents in digital formate so that user can easily access documents. governments organisation also find difficulty for handling users documents it also help to reduce there problem

\section{REFRENCES}

1) Prof. Masuda Isaeva "Paperless UniversityHow we can make it work?" Published in: 2016 15th International Conference on Information Technology Based Higher Education and Training (ITHET)

2) Rupali Patil; R. G. Karandikar, "Digitization of Documented Signals Using Vertical Scanning" Published in: 2015 International Conference on Microwave, Optical and Communication Engineering (ICMOCE) Date of Conference: 18-20 Dec. 2015

3) Owczarek, Wojciechowski, Murlewski, Sakowicz, Napieralski "ELECTRONIC DOCUMENT MANAGEMENT SYSTEM" Published in: Proceedings of the International Conference Mixed Design of Integrated Circuits and System, 2006. MIXDES 2006. Date of Conference: 22-24 June 2006

4. Jinal Shah; Sukumar Gaonkar; Aashika Shetty; Radha Shankarmani "Digitization and Paperless Processing through the use of mobile imaging Technology" Published in: 2016 International Conference on Circuit, Power and Computing Technologies (ICCPCT) Date of Conference: 18-19 March 2016

5. L. Diesendruck, L. Marini, R. Kooper, M. Kejriwal, and K. McHenry "A Framework to Access Handwritten Information within Large Digitized Paper Collections" A framework to access handwritten information within large digitized paper collections Publication Type Conference Paper Year of Publication 2012 